\title{
A water extract of Malva verticillata seeds suppresses osteoclastogenesis and bone resorption stimulated by RANK ligand
}

Ki-Shuk Shim, Chung-Jo Lee, Nam-Hui Yim, Hyunil Ha and Jin Yeul Ma*

\begin{abstract}
Background: Malva verticillata seeds are used as a therapeutic medicine to treat kidney dysfunction in traditional Chinese medicine (TCM). TCM has suggested that herbal medicine tonifying kidney function may have beneficial effect on bone metabolism.

Methods: Osteoclastogenesis was examined in bone marrow macrophages by measuring tartrate-resistant acid phosphatase (TRAP) activity and counting the number of TRAP-stained multinuclear cells. The activation of receptor activator of nuclear factor-kB (RANK) ligand signaling, and the expression of c-Fos and nuclear factor of activated T-cells, cytoplasmic 1 (NFATc1) were investigated by western blot analysis. Transcription factor and bone resorption marker mRNA levels were evaluated using real-time quantitative polymerase chain reaction. The bone resorption activity of mature osteoclast was examined in osteoclasts cultured on a hydroxyapatite-coated culture plate.

Results: A water extract of M. verticillata seeds (WEMV) inhibited osteoclastogenesis stimulated by RANKL. WEMV also strongly inhibited expression of c-Fos and NFATc1 as well as phosphorylation of c-Jun N-terminal kinase, p38, $\mathrm{I}-\mathrm{kBa}$, and phospholipase $\gamma 2$. Furthermore, WEMV significantly attenuated osteoclast resorption activity and downregulated mRNA expression of resorption markers.
\end{abstract}

Conclusion: These results demonstrate that WEMV inhibits osteoclastogenesis and bone resorption by suppressing the RANKL signaling pathway and suggest that $M$. verticillata seeds may be used as a therapeutic candidate in complementary alternative medicine to treat pathological bone diseases.

Keywords: Malvaceae, Malva verticillata seeds, Receptor activator of nuclear factor-kB ligand, Osteoclasts, Nuclear factor of activated T-cells, cytoplasmic 1

Abbreviations: ALP, Alkaline phosphatase; AP-1, Activator protein 1; BCA, Bicinchoninic acid assay; BMC, Bone marrow cell; BMM, Bone marrow macrophages; BMP-2, Bone morphogenetic protein-2; CA-NFATc1, Constitutively expressed form of NFATC1; CREB, CAMP response element binding; FBS, Fetal bovine serum; GC/MS, Gas chromatography/mass spectrometry; GFP, Green fluorescent protein; HPRT, Hypoxanthine phosphoribosyltransferase; IACUC, Institutional animal care and use committee; Id2, Inhibitor of DNA binding 2; JNK, c-Jun N-terminal kinase; MafB, v-Maf Avian musculoaponeurotic fibrosarcoma oncogene homolog B; MAPK, Mitogen-activated protein kinase; M-CSF, Macrophage colony stimulating factor; MNCs, Multinuclear osteoclasts; NFATc1, Nuclear factor of activated T-cells, cytoplasmic 1; NF-kB, Nuclear factor-kappaB; NIST, National Institute of Standards and Technology; PBS, Phosphate-buffered saline; PLC, Phospholipase C; PVDF, Polyvinylidene fluoride; qRT-PCR, Real-time quantitative polymerase chain reaction; RANKL, Receptor activator of nuclear factor-kB ligand; RIPA, Radioimmunoprecipitation assay; TCM, Traditional Chinese medicine; TRAP, Tartrate-resistant acid phosphatase; WEMV, Water extract of M. verticillata seeds.

\footnotetext{
* Correspondence: jyma@kiom.re.kr

KM Application Center, Korea Institute of Oriental Medicine, 70 Cheomdanro

Dong-gu, Daegu 41062, Republic of Korea
} 


\section{Background}

Osteoporosis is a skeletal disorder characterized by low bone mineral density and deteriorated bone microstructure, which increases the incidence of bone fracture. It is caused mainly by an imbalance between bone resorption and bone formation when an increased rate of bone resorption overcomes a decreased rate of bone formation caused by estrogen deficiency, aging, or an inadequate intake of calcium or vitamin D3, especially in elderly populations [1, 2]. Receptor activator for nuclear factor$\mathrm{kB}$ (RANK) ligand (RANKL) is a cytokine that is essential for osteoclast differentiation, regulation of osteoclast bone resorbing activity and osteoclast apoptosis [3]. RANKL activates various signaling molecules and critical transcription factors in osteoclastogenesis such as mitogen-activated protein kinase (MAPK) and nuclear factor of activated T-cells, cytoplasmic 1 (NFATc1). Recent studies have investigated the molecular mechanism of osteoclastogenesis and bone resorption to develop anti-osteoporosis agents with fewer undesirable side effects $[4,5]$. Herbal medicine and natural products have recently been suggested to mitigate bone loss and ameliorate osteoporosis with fewer side effects through their ability to affect bone cells [6-8].

Malva verticillata Linn is a species of the mallow genus Malva in the family of Malvaceae [9]. Seeds of $M$. verticillata, commonly known as Dong Kui $\mathrm{Zi}$ or Donggyuja, have been used to enhance kidney strength, remove renal stones, and treat chronic mastitis and hypogalactia in traditional Chinese medicine (TCM) $[9,10] . M$. verticillata seeds contain various polysaccharides and flavonoids with a number of pharmacological activities including hypoglycemic activity [11-13]. In TCM, herbal medicine is used to invigorate kidney function so as to strengthen musculoskeletal organs such as bone [14, 15]. Chinese herbal formulas or herbal medicines prescribed in TCM to nourish or replenish kidney function have been shown to have a positive effect on ovariectomy-induced osteoporosis [16] and a pharmaceutical effect on bone by regulating osteoclast differentiation [17] or mast cell activity [18]. Based on the results of these studies, M. verticillata seeds used in TCM to stimulate kidney function might have a beneficial effect on bone by regulating bone cell differentiation; however, no study has explored the effect of $M$. verticillata seeds on bone cell differentiation and/or function.

In this study, we examined whether a water extract of $M$. verticillata seeds (WEMV) exerts a pharmaceutical effect to regulate osteoclastogenesis or osteogenesis. We found that WEMV inhibited RANKL-induced osteoclastogenesis, but WEMV had no effect on bone morphogenetic protein-2 (BMP-2)- or ascorbic acid/ $\beta$ glycerophosphate-induced osteogenesis. To increase our understanding of the molecular mechanism underlying
WEMV activity in osteoclastogenesis and bone resorption, we investigated the effect of WEMV on RANKL signaling and the expression of genes involved in bone resorption.

\section{Methods \\ Preparation of a water extract of $M$. verticillata seeds}

$M$. verticillata seeds (Yeongcheon herb, Korea) were authenticated by Prof. K.H. Bae (Chungnam National University, Korea). A voucher specimen with registration number 221 is maintained in the herbarium of the KM Application Center at the Korea Institute of Oriental Medicine. Dried M. verticillata seeds (50 g) were extracted by boiling in distilled water $(1 \mathrm{~L})$ for $3 \mathrm{~h}$ as described previously [19]. The water extract of $M$. verticillata seeds (WEMV) was filtrated using standard sieves $(150 \mu \mathrm{m})$, concentrated by lyophilization, and stored at $-20{ }^{\circ} \mathrm{C}$ until use. A WEMV stock solution was prepared by dissolving the lyophilized powder in distilled water followed by filtering through a $0.2 \mu \mathrm{m}$ syringe filter. The yield of dried extract from the staring materials was approximately $8.96 \%(\mathrm{w} / \mathrm{w})$.

\section{Gas chromatography/ mass spectrometry analysis}

The lyophilized water extract of $M$. verticillata seeds was submitted to separation through gas chromatography with detection by mass spectrometry (GC/MS). The analysis was performed in a GC/MS system (Agilent Technologies, Atlanta, GA, USA) using DB-5 MS capillary column $(30 \mathrm{~m} \times 0.25 \mathrm{~mm} \times 0.25 \mu \mathrm{m})$. Chromatographic conditions were as follows: the extract $(1 \mu \mathrm{L})$ was injected in split mode with a ratio of $1 / 20$ at $350{ }^{\circ} \mathrm{C}$, oven initial temperature was $70{ }^{\circ} \mathrm{C}$ during $1 \mathrm{~min}$, followed by heating at a rate of $5{ }^{\circ} \mathrm{C} / \mathrm{min}$ at $300{ }^{\circ} \mathrm{C}$, and totally run for $67 \mathrm{~min}$. The mass analyzer was set to scan from 10 to 800 atomic mass unit. $\beta$-sitosterol was used as standard marker for GC/MS analysis. Peak identification was carried out comparison of the experimental mass spectrum in the National Institute of Standards and Technology (NIST) and Wiley GC-MS libraries. Fourteen components, including 1,3-dihydroxyacetone dimer, d-alanine, 5-hydroxymethyl furfural, 2-hydroxygamma-butyrolactone, palmitic acid, oleamide, and $\beta$ sitosterol, were identified in WEMV (data not shown).

\section{Cell culture}

Mouse bone marrow macrophages (BMMs) were obtained from mouse bone marrow cells (BMCs) as described previously [20]. For osteoclast differentiation, BMMs $\left(1 \times 10^{4}\right.$ cells/well, 96-well plate $)$ were cultured in $\alpha$-MEM differentiation medium containing $60 \mathrm{ng} / \mathrm{mL}$ of macrophage colony stimulating factor (M-CSF) and $100 \mathrm{ng} / \mathrm{mL}$ of RANKL for 4 days. Tartrate-resistant acid phosphatase (TRAP)-positive stained cells with more than three nuclei were counted as multinuclear osteoclasts 
[TRAP (+) MNCs]. For osteoblast differentiation, C2C12 cells (ATCC, USA) were cultured $\left(1 \times 10^{4}\right.$ cells/well, 96well plate) in $\alpha$-MEM differentiation medium containing $100 \mathrm{ng} / \mathrm{mL}$ of BMP-2 for 3 days. Primary calvarial osteoblasts $\left(5 \times 10^{4}\right.$ cells/well, 48-well plate) obtained as described previously [21] were cultured in $\alpha$-MEM differentiation medium containing $50 \mu \mathrm{g} / \mathrm{mL}$ ascorbic acid and $10 \mathrm{mM} \beta$-glycerophosphate for 5 days. BMMs were cultured in $\alpha$-MEM proliferation medium, and $\mathrm{C} 2 \mathrm{C} 12$ cells and osteoblasts were cultured in DMEM proliferation medium containing various concentrations of WEMV for 2 days to examine cytotoxicity using a CCK-8 assay (Dojindo Molecular Technologies Inc., Tokyo, Japan). All media contained $10 \%$ fetal bovine serum (FBS) and antibiotics $(100 \mathrm{U} / \mathrm{mL}$ penicillin and $100 \mu \mathrm{g} / \mathrm{mL}$ streptomycin) and were replaced at 3-days intervals.

\section{Enzyme assay}

TRAP and alkaline phosphatase (ALP) activity assay were performed as described previously [20]. Briefly, cells were fixed in $10 \%$ formalin, permeabilized with $0.1 \%$ Triton X-100, and incubated with TRAP assay buffer $(50 \mathrm{mM}$ sodium tartrate, $0.12 \mathrm{M}$ sodium acetate, $\mathrm{pH} 5.2)$ with $p$-nitrophenyl phosphate $(1 \mathrm{mg} / \mathrm{mL})$ or incubated with ALP assay buffer $[100 \mathrm{mM}$ sodium carbonate (pH 10), $\left.1 \mathrm{mM} \mathrm{MgCl} 2,50 \mathrm{mM} \mathrm{Na}_{2} \mathrm{CO}_{3}\right]$ with naphthol AS-BI phosphate. After a 10-min incubation at $37{ }^{\circ} \mathrm{C}$, the reaction was stopped with stop solution and the absorbance was measured. MNCs were stained with TRAP staining buffer containing naphthol AS-MX phosphate and Fast Red Violet LB (Sigma-Aldrich).

\section{Western blot analysis}

Cells were washed twice with phosphate-buffered saline (PBS) and total cell lysate was obtained using Radioimmunoprecipitation assay (RIPA) buffer (Millipore, MA, USA) containing protease and phosphatase inhibitors. Protein concentration was determined using a bicinchoninic acid assay (BCA) Assay Kit (Thermo Scientific, Rockford, IL, USA). Total protein $(30 \mu \mathrm{g})$ was separated by $12.5 \%$ SDSPAGE gel electrophoresis, transferred to polyvinylidene fluoride (PVDF) membrane, and immunoblotted with specific antibody. Antibodies for MAPK, nuclear factorkappaB (NF-kB), or phospholipase C (PLC) signaling proteins (Cell Singling Technology, MA, USA) or c-Fos and NFATc1 transcription factors (Santa Cruz Biotechnology, CA, USA) were used in this study. Chemiluminescent signals were detected using a ChemiDoc imaging system (Bio-Rad Laboratories, CA, USA) and a chemiluminescence reagent (Thermo Scientific).
Real-time quantitative polymerase chain reaction (qRT-PCR) Total RNA was isolated using an RNAspin total RNA extraction kit (Intron, Daejeon, Korea). cDNA was synthesized from $1 \mu \mathrm{g}$ of total RNA using AccuPower RT-PreMix (Bioneer, Daejeon, Korea) according to the manufacturer's protocol. qRT-PCR analysis was performed using a CFX96 Touch Real-Time PCR System (Bio-Rad, CA, USA) and AccuPower GreenStar qPCR Master Mix (Bioneer, Daejeon, Korea). The qRT-PCR reactions comprised 40 cycles at $94{ }^{\circ} \mathrm{C}$ for $20 \mathrm{~s}$ and $60{ }^{\circ} \mathrm{C}$ for $40 \mathrm{~s}$. All reactions were performed in triplicate. The relative expression levels of target genes were normalized to hypoxanthine phosphoribosyltransferase (HPRT) expression and analyzed using Bio-Rad CFX Manage 3.1 software (Bio-Rad, CA, USA).

\section{Retrovirus preparation and infection}

A pMX-puro-green fluorescent protein (GFP) retrovirus vector (provided by Dr. T. Kitamura, University of Tokyo) and a pMX-Ca-NFATc1 vector encoding HAtagged Ca-NFATc1, a constitutively expressed form of NFATc1 provided by Dr. N.A. Clipstone (Northwestern University), were used for retrovirus preparation as described previously [20]. Briefly, to generate retroviral stocks, the vector was transfected into Plat-E retroviral packaging cells. BMMs $\left(3 \times 10^{6}\right.$ cells, $100-\mathrm{mm}$ dish $)$ were infected with retrovirus soup from the packaging cells by incubation with polybrene $(6 \mu \mathrm{g} / \mathrm{mL})$ for $8 \mathrm{~h}$. Infected BMMs were selected with puromycin $(2 \mu \mathrm{g} / \mathrm{mL}$, Sigma-Aldrich) for 5 days. The selected BMMs were cultured in differentiation medium with WEMV for 4 days and then stained with TRAP-staining solution.

\section{Bone resorption assay}

Osteoclasts obtained from a BMCs-osteoblasts coculture system were cultured on OsteoAssay Surface plates (Corning, MA, USA). Osteoclasts were preincubated with WEMV for $3 \mathrm{~h}$ and further cultured in differentiation medium for an additional $16 \mathrm{~h}$. After removing cells, stained osteoclasts and resorbed areas were observed under an inverted microscope (40x magnification). The resorption areas were analyzed in three randomly selected fields of each well using Image J software.

\section{Statistical analysis}

Statistical significance of the differences in TRAP activity, MNC number, and mRNA levels of genes was analyzed using Student's $t$-test. Data are presented as means \pm the standard deviation of three independent experiments. Differences are considered significant at $p<0.05$. 


\section{Results}

WEMV inhibits RANKL-induced osteoclast differentiation in BMMs

We first examined the pharmaceutical effect of WEMV on osteoclast differentiation. BMMs were cultured in differentiation medium with or without WEMV for 4 days. As shown in Fig. 1a, RANKL induced differentiation of BMMs into TRAP-positive stained MNCs; however, WEMV inhibited RANKL-induced TRAP activity and MNC formation $(p<0.01$ ) (Fig. 1b and c) in a dose-dependent manner. WEMV at a concentration of $160 \mu \mathrm{g} / \mathrm{mL}$ almost completely suppressed the MNC number $(9 \pm 2, p<0.01)$ without inducing cytotoxicity compared with the control $(301 \pm 11)$ (Fig. 1d) suggesting that inhibitory activity of WEMV was specific for osteoclastogenesis. However, because osteoclastogenesis involves sequential steps in the differentiation of precursor cells into pre-osteoclast and osteoclasts, the stage of osteoclastogenesis that was inhibited by WEMV was unclear. To determine the effect of WEMV on each stage of osteoclastogenesis, BMMs were cultured with WEMV $(160 \mu \mathrm{g} / \mathrm{mL})$ at four time points after which MNC numbers were counted on day 4 . We found that the MNC number was suppressed significantly when WEMV was added on day $0(2 \pm 1, p<0.01)$ or on day 1 $(203 \pm 2, p<0.01)$ as compared with the control $(275 \pm$ 22) (Fig. 1e), but MNC formation was not inhibited by WEMV when added on days 2 or 3 suggesting that WEMV inhibits early osteoclast differentiation.

\section{WEMV does not affect osteoblast differentiation}

BMP-2 and ascorbic acid/ $\beta$-glycerophosphate induce osteoblast differentiation accompanied by an increase in differentiation markers such as ALP. We examined whether WEMV has any effect on osteoblast differentiation of $\mathrm{C} 2 \mathrm{C} 12$ or calvarial osteoblast cells by measuring ALP activity. We found that WEMV did not increase BMP-2-induced ALP activity or decrease the viability of $\mathrm{C} 2 \mathrm{C} 12$ cells, even at high concentration $(160 \mu \mathrm{g} / \mathrm{mL})$ (Fig. $2 \mathrm{a}$ and b). We also found that WEMV did not affect osteoblast differentiation or viability of calvarial cells (Fig. 2c and d). These results suggested that WEMV does not regulate the early stage of osteoblast differentiation and proliferation.

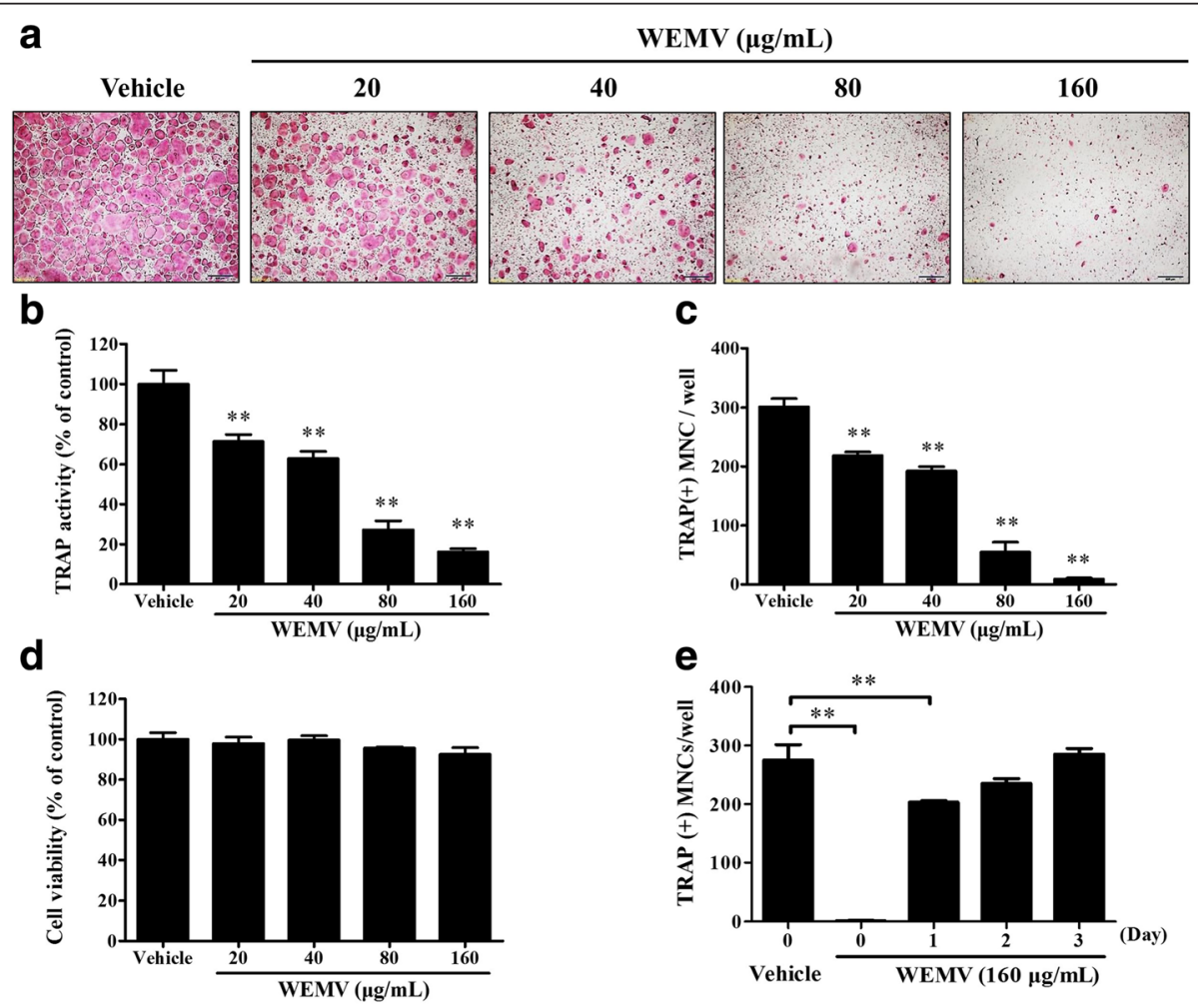

Fig. 1 WEMV has a negative effect on osteoclastogenesis. BMMs were cultured for 4 days in differentiation medium containing WEMV at concentrations of 20,40,80, and $160 \mu \mathrm{g} / \mathrm{mL}$. a TRAP-positive stained cells were visualized using TRAP staining solution. b TRAP activity was measured by TRAP assay with p-nitrophenyl phosphate as described in the Methods. c TRAP-positive stained MNCs were counted under an inverted microscope (40× magnification). d Cell viability percentage were determined using a CCK assay after a 2-day incubation with the indicated concentrations of WEMV. e BMMs were cultured in differentiation medium with WEMV $(160 \mu \mathrm{g} / \mathrm{mL})$ for the indicated numbers of days. TRAP-positive stained MNCs were counted on day $4 .{ }^{* *} p<0.01$ versus control 

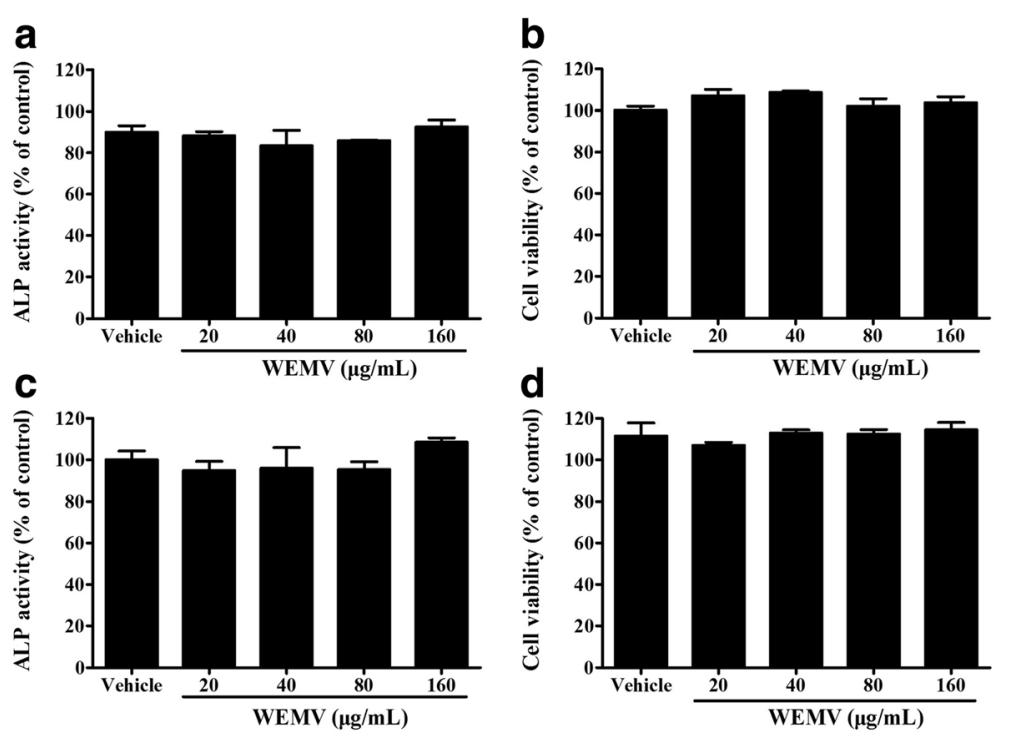

Fig. 2 WEMV does not affect osteoblast differentiation. C2C12 cells were cultured in differentiation medium with the indicated concentrations of WEMV for 3 days. a ALP activity of C2C12 cells was determined using an ALP assay with naphthol AS-BI phosphate as described in the Methods. b A CCK-8 assay was performed to determine the viability of $\mathrm{C} 2 \mathrm{C} 12$ cells after a 2-day incubation with the indicated concentrations of WEMV. Calvarial osteoblast cells were cultured in differentiation medium for 15 days. c ALP activity and (d) calvarial osteoblast cell viability were examined after 15- and 2-day incubations, respectively

WEMV suppresses NFATc1 and c-Fos expression in BMMs To determine how WEMV suppresses osteoclastogenesis, we investigated c-Fos and NFATc1 expression. BMMs pretreated with WEMV and control were further incubated in osteoclast differentiation medium for various time periods. c-Fos and NFATc1 mRNA and protein levels increased markedly on days 1 and 2 in response to RANKL stimulation (Fig. 3a and b), but WEMV almost completely abolished their protein levels. WEMV reduced c-Fos mRNA level to $63 \%$ on day 1 and NFATc1 mRNA level to $22 \%$ on day $2(p<0.01)$ relative to control. Since NFATc1 is the master transcription factor that regulates osteoclastogenesis, we investigated whether ectopic NFATc1 expression could restore osteoclastogenesis that was suppressed by WEMV. BMMs were infected with control retroviral vector or a recombinant retroviral vector harboring a CA-NFATc1 and were then cultured under osteoclast differentiation conditions. WEMV completely inhibited MNC formation in BMMs infected with the control vector, but did not inhibit MNC formation in BMMs infected with CA-NFATc1 (Fig. 3c). As a counterpoint to the effect of WEMV on NFATc1-inducing factors such as c-Fos, we investigated the mRNA expression of $\mathrm{v}$-Maf avian musculoaponeurotic fibrosarcoma oncogene homolog $\mathrm{B}$ (MafB) and inhibitor of DNA binding 2 (Id2), which are transcriptional repressors of NFATc1 in BMMs. WEMV significantly attenuated RANKL-induced downregulation of MafB mRNA level to $76 \%$ and increased Id2 mRNA level by two fold on day $1(p<0.01)$ relative to the control (Fig. 3b). These results indicated that the effect of WEMV on osteoclastogenesis may involve the inhibition of NFATc1 expression.

\section{WEMV inhibits MAPK, NF-kB, and PLC 2 activation in BMMs}

RANKL-RANK interaction activates early signaling pathways, including MAPK and NF-kB signaling, to regulate NFATc1 expression during osteoclastogenesis. We further explored WEMV inhibition of early-stage of osteoclast differentiation by examining the effect of WEMV on RANKL-induced activation of MAPK and NF-kB signaling. As shown in Fig. 4, RANKL increased c-Jun N-terminal kinase (JNK) and p38 phosphorylation markedly (lane 3 ) as well as I-kB $\alpha$ phosphorylation (lane 2) and degradation (lane 3). However, WEMV decreased JNK phosphorylation (lane 7) and p38 phosphorylation (lane 7) at $15 \mathrm{~min}$ relative to the control. In addition, WEMV partially reduced I-kB $\alpha$ phosphorylation (lane 6) at $5 \mathrm{~min}$ and impaired I-kB $\alpha$ degradation (lane 7 and 8) at 15 and $30 \mathrm{~min}$ relative to the control. We next evaluated whether WEMV affects Src-PLC-Ca ${ }^{2+}$ pathway to regulate NFATc1 expression. WEMV markedly suppressed RANKL-induced PLC 2 phosphorylation (lane 6) at $5 \mathrm{~min}$ and Src phosphorylation (lane 7) at $15 \mathrm{~min}$.

\section{WEMV inhibits bone resorption activity of osteoclasts}

To examine WEMV activity on bone resorption in a physiologically relevant manner, mature osteoclasts obtained from a BMCs-osteoblasts coculture system were 

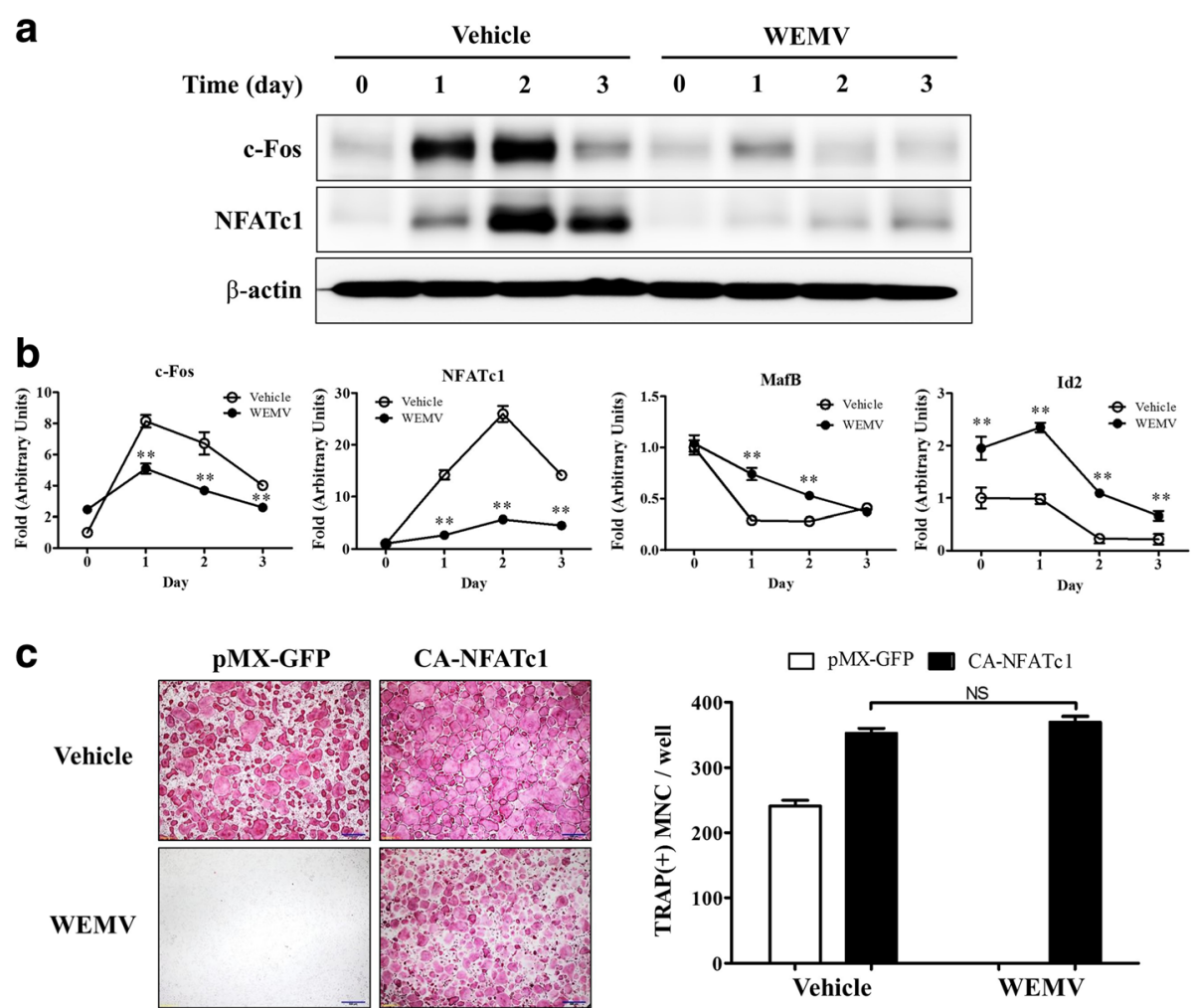

Fig. 3 WEMV downregulates C-Fos and NFATc1 expression. BMMs were pretreated with WEMV (160 $\mathrm{gg} / \mathrm{mL})$ for $3 \mathrm{~h}$ and cultured in differentiation medium for the indicated times as described in the Methods. Total RNA or protein was obtained at the indicated time points. a Total cell lysates $(30 \mathrm{\mu g})$ were separated by SDS-PAGE electrophoresis and transferred to PVDF membranes. Western blot analysis was performed with antibodies specific for c-Fos (top), NFATc1 (middle), and $\beta$-actin (bottom). b qRT-PCR analysis of c-Fos, NFATc1, MafB, and Id2 was performed at the indicated time points. Each mRNA level was normalized to that of HPRT. c BMMs were infected with retrovirus vector harboring GFP (control) or CA-NFATc1 in the presence of polybrene $(6 \mu \mathrm{g} / \mathrm{mL})$ for $8 \mathrm{~h}$. The infected cells were cultured in a-MEM medium containing puromycin $(2 \mu \mathrm{g} / \mathrm{mL})$ for 5 days and then cultured in differentiation medium containing WEMV $(160 \mu \mathrm{g} / \mathrm{mL})$ for 4 days. TRAP-positive stained MNCs were counted after TRAP staining under an inverted microscope (40× magnification). ${ }^{* *} p<0.01$ versus control. NS; not significance
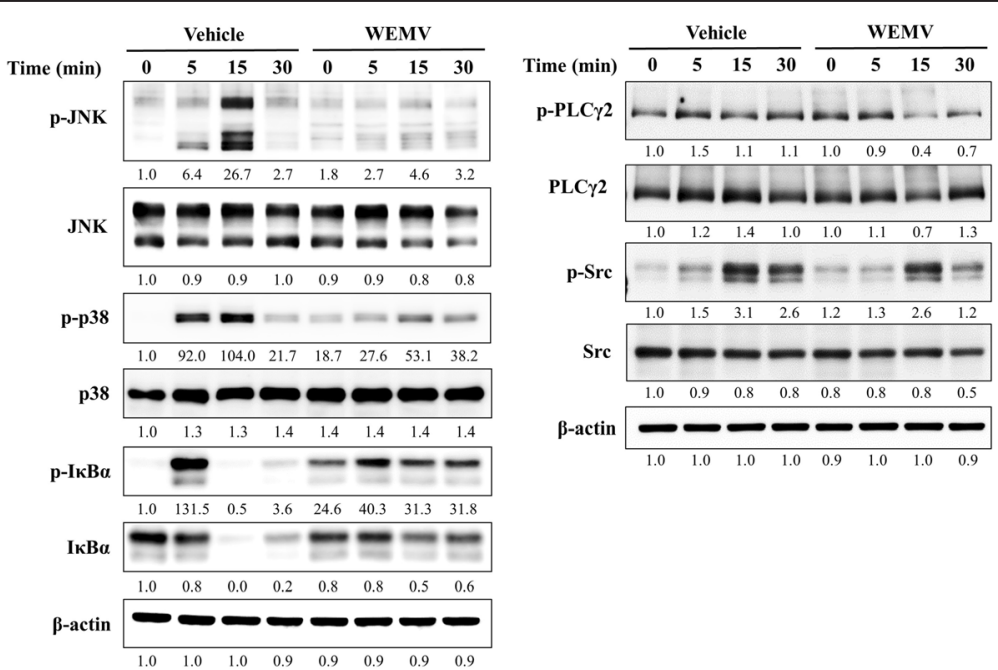

Fig. 4 WEMV suppresses MAPK, NF-kB, and PLC $y$ signaling pathways. BMMs pretreated with WEMV (160 $\mu \mathrm{g} / \mathrm{mL})$ for $3 \mathrm{~h}$ were exposed to RANKL for the indicated times. Total cell lysate $(30 \mu \mathrm{g} / \mathrm{mL})$ were separated by SDS-PAGE electrophoresis, transferred to PVDF membrane, and immunoblotted with antibodies specific for each signaling pathway 
cultured with or without WEMV on a hydroxyapatitecoated plate. As shown in Fig. 5a, RANKL treatment stimulated mature osteoclasts to resorb on the plate. However, WEMV substantially decreased the resorption area without affecting the MNC number (Fig. 5b and c). Quantitative analysis of the resorption area demonstrated that WEMV concentrations of $80 \mu \mathrm{g} / \mathrm{mL}$ and $160 \mu \mathrm{g} / \mathrm{mL}$ significantly suppressed bone resorption activity of osteoclasts to $12.56 \pm 1.86$ and $12.49 \pm 1.37 \%$ $(p<0.05)$, respectively, relative to the control $(17.46 \pm$ $1.07 \%)$. To delineate the molecular mechanism underlying the effect of WEMV on bone resorption, we examined whether WEMV affects the expression of TRAP, ATPv0d2, and cathepsin $K$, all of which are involved in bone resorption. We found that WEMV caused marked downregulation of the mRNA levels of TRAP, ATP6v0d2, and cathepsin $\mathrm{K}$ to 25,39 , and $20 \%(p<0.01)$, respectively, relative to the control (Fig. 5d).

\section{Discussion}

$M$. verticillata seeds have been used in Asia for the treatment of nephrolithiasis and cystolithiasis and for enhancing kidney strength and reducing lower back and bladder pain. The aim of this study was to evaluate the pharmaceutical effect of WEMV on osteoclastogenesis and osteogenesis. We showed that WEMV inhibited osteoclastogenesis by decreasing NFATc1 expression and inhibited bone resorption by downregulating the expression of resorption markers, albeit at high concentration.

We found that WEMV suppressed osteoclast differentiation (Fig. 1) and NFATc1 expression significantly (Fig. 4). NFATc1 plays a crucial role in the regulation of osteoclast transcription during osteoclastogenesis. Upon RANKL stimulation, initiation and/or amplification of NFATc1 expression is achieved mainly by the activation of MAPK, NF-kB, and PLCy-activated $\mathrm{Ca}^{2+}$ signaling pathways [22] which subsequently activate key transcription factors, activator protein 1 (AP-1), NFATc1, and cAMP response element binding (CREB) [3]. These RANKL-stimulated pathways are somewhat sequential with respect to NFATc1 induction, but they cooperate in the amplification of NFATc1 expression to regulate and facilitate osteoclast-specific transcription. Our results showed that WEMV inhibited the activation of MAPK,
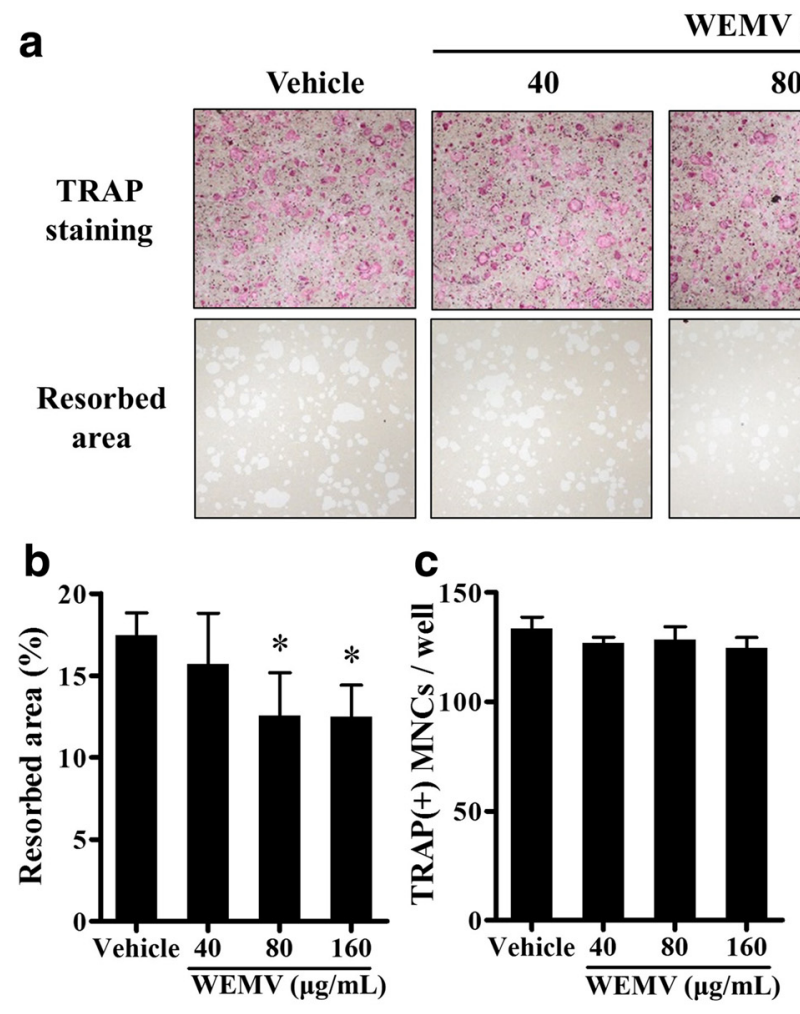

\section{$\operatorname{WEMV}(\mu \mathrm{g} / \mathrm{mL})$}

80
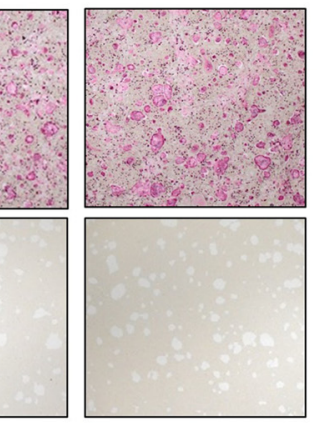

d

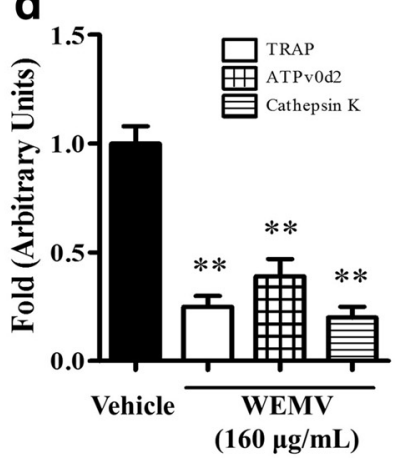

Fig. 5 WEMV inhibits bone resorption. Osteoclasts with bone resorbing function were obtained from coculture of BMCs and calvarial osteoblasts. The cells were seeded on an OsteoAssay Surface plate and cultured in differentiation medium containing WEMV (160 $\mu \mathrm{g} / \mathrm{mL}$ ) for $16 \mathrm{~h}$. a MNCs (upper panel) or the resorbed areas (lower panel) were photographed under an inverted microscope (40× magnification). $\mathbf{b}$ The percentage of resorbing areas on the plate was quantified using Image J software. c TRAP-positive stained MNCs were counted to evaluate the cytotoxic effect of WEMV on mature osteoclasts. $\mathbf{d}$ The mRNA levels of bone resorption genes (TRAP, ATPv0d2, and cathepsin K) were analyzed by $q R T-P C R .{ }^{*} p<0.05 ;{ }^{* *} p<0.01$ versus control 
NF-kB, and PLC $\gamma$ signaling as well as suppressed c-Fos expression significantly (Figs. 3 and 4), suggesting that WEMV inhibition to the upstream signaling cascade of NFATc1 suppresses NFATc1 expression and osteoclastogenesis. Regarding distinct activation of these signaling pathways for NFATc1 expression, different inhibitory components present in WEMV might affect each signaling pathway during osteoclastogenesis.

WEMV attenuated RANKL-induced downregulation of MafB and Id2 significantly at days 1 and 2 accompanied by suppression of NFATc1 expression (Fig. 3). Transcription repressors, such as MafB and Id2, are expressed abundantly in BMMs to interrupt the formation of transcriptional complex or the ability of complexes to transcriptionally regulate NFATc1 expression thereby preventing non-specific initiation of osteoclastogenesis and maintaining the potential of BMMs to differentiate [23]. In response to RANKL or TNF $\alpha$ stimulation, the expression of transcription repressors is downregulated markedly within $48 \mathrm{~h}$; however, their ectopic expression blocks osteoclastogenesis [24, 25]. Thus, WEMV activity to maintain the expression of transcription repressor may partially contribute to suppress NFATc1 expression and thus block osteoclastogenesis, although RANKL stimulates osteoclast precursors.

Bone resorption requires high levels of resorption markers such as TRAP, ATPv0d2, and cathepsin $K$, in mature osteoclasts to degrade bone matrix. Inhibition of bone resorption markers such as cathepsin $\mathrm{K}$ results in decreased resorption activity by disrupting the intracellular vesicle transport system that delivers degraded collagen [26]. Our results showed that WEMV suppressed either bone resorption or transcription of all of the above marker genes significantly in osteoclasts without affecting the MNC number (Fig. 5), suggesting that WEMV may block either generation of resorbing protein or vesicular trafficking system for bone resorption. With respect to the inhibition of bone resorption and osteoclastogenesis by WEMV with no effect on osteoblast differentiation or proliferation, an evaluation of the antiosteoporosis potential of WEMV alone or in combination with TCM stimulating osteogenesis in an in vivo animal model system representing pathological bone disorders would be of interest for a future study.

$M$. verticillata seeds contains several chemical constituents, such as polysaccharides, fatty acid, flavonoid, terpenes, and sterol [10]. Different polysaccharides, such as MVS-I, MVS-IIA, or MVS-V, has been identified as major polysaccharide in water extract of $M$. verticillata seeds [27, 28]. Among them, arabinogalactan, a part component of MVS-I or MVS-IIA, weakly inhibits osteoclast differentiation of RAW264.7 cells (13.8 \% inhibition at $100 \mu \mathrm{g} / \mathrm{ml}$ ) by downregulating the expression of TRAP and cathepsin K [29]. However, peptidoglycan, other polysaccharides identified in water extract of $M$. verticillata seeds, is known to have stimulatory effect on osteoclast differentiation as well as bone resorption [30], which should be avoided for anti-osteoclast agents. Besides polysaccharide, $M$. verticillata seeds also have oil constituents (11\% of total yield) including $16.18 \%$ palmitic, $7.7 \%$ oleic, $61.6 \%$, linoleic, and $7.9 \%$ stearic acids [10]. We also identified the presence of fatty acid (palmitic acid and oleamide) and sterol ( $\beta$-sitosterol) in WEMV by GC/MS analysis (data not shown). Regarding the inhibitory activity of fatty acid and sterol on osteoclast differentiation, palmitic acid (50\% inhibition at $10 \mu \mathrm{g} / \mathrm{ml}$ ) inhibits osteoclastogenesis by suppressing the expression of fatty acid binding receptor, while oleamide (150\% inhibition at $100 \mu \mathrm{M})$ suppresses bone resorption by inhibiting gap junction of osteoclasts [31, 32]. In addition, several terpenes and sterol ( $\beta$-sitosterol; $15 \%$ inhibition at $1 \mu \mathrm{M}$ ) inhibit osteoclast differentiation by inhibiting TRAP activity although the possible action mechanism is unknown [33]. Thus, considering the inhibitory activity of WEMV at high concentration, but less amounts of identified components present in $M$. verticillata seeds, it suggest that the inhibitory effect of WEMV might result from the combined effect of these chemical constituents such as polysaccharide, fatty acid or sterol in WEMV rather the action of individual constituents on osteoclast differentiation and resorption. Since we identified the presence of some chemical constituents as marker component for standardization of WEMV (data not shown), further studies to characterize the chemical properties of WEMV, to identify the unknown active components in WEMV, and to elucidate its action mechanism on osteoclast differentiation are needed to understand the effect of WEMV on osteoclasts and the action mechanism.

WEMV specifically inhibited the osteoclast differentiation potential of BMMs by inhibiting the RANKL signaling pathway. In TCM, $M$. verticillata seeds has been used to improve flow between the nutrient phase and the defense phase that enhances kidney function. Kidney has a function storing an essence of life, known as kidney essence, which generates and nourishes bone marrow to make a fullness of marrow in bone, forcefulness of musculoskeletal organ, and hardness of bone [14]. When kidney essence is exhausted by aging or disease, kidney function to provide the nutrition in bone marrow is insufficient to support bone microenvironment that deteriorates musculoskeletal function and increases pain in musculoskeletal organ, which is similar to manifestations observed in pathological bone diseases, such as osteoporosis and rheumatoid arthritis, caused by an imbalance of bone remodeling. Several studies have reported that herbs or herbal formulas originally used in TCM to tonify kidney function have positive effects on 
bone metabolism by directly inhibiting osteoclast differentiation [17] or resorption [34] or by regulating osteoblast-dependent osteoclastogenesis [35]. Regulation of the RANKL signaling on osteoclastogenesis has been suggested as one of the mechanisms by which herbal medicines tonifying kidney essence affect bone metabolism [36]. Thus, WEMV inhibition of the RANKL signaling pathway and bone resorption may be the molecular mechanism by which WEMV affects bone metabolism in addition to its pharmaceutic effect to strengthen kidney function in TCM.

\section{Conclusions}

We have demonstrated that WEMV caused marked inhibition of osteoclastogenesis by suppressing the RANKL signaling axis and bone resorbing function by downregulating bone resorption markers. This study reveals the molecular mechanism underlying the effect of WEMV on osteoclastogenesis and suggests that WEMV may be a valid component of therapeutic prescriptions used in TCM to treat bone diseases caused by excess osteoclast differentiation and bone resorption.

\section{Acknowledgment}

This research was supported by a grant (K15280) funded by the Korea Institute of Oriental Medicine. We were thankful to Dr. T. Kitamura and Dr. N.A. Clipstone (Northwestern University) for the reagents.

\section{Availability of data and materials}

All data and materials are contained and described within the manuscript.

\section{Authors' contributions}

KSS and JYM designed the study and wrote the manuscript. CJL and NHY carried out the experiments. HHH analyzed the data. All authors read and approved the final manuscript.

\section{Competing interests}

The authors declare that they have no competing interests.

\section{Consent for publication}

Not applicable. This article is not a clinical study involving human participants and this manuscript does not contain any individual clinical data.

\section{Ethics approval and consent to participate}

Animal experiments were reviewed and approved by the Institutional Animal Care and Use Committee at the Korea Institute of Oriental Medicine (Approval number of animal experiment, 15-006) and conducted according to the National Institute of Health's Guidelines for the Care and Use of Laboratory Animals followed the National Animal Welfare Law of Korea.

Received: 24 September 2015 Accepted: 17 August 2016 Published online: 31 August 2016

\section{References}

1. Riggs BL, O'Fallon WM, Muhs J, O'Connor MK, Kumar R, Melton 3rd LJ. Long-term effects of calcium supplementation on serum parathyroid hormone level, bone turnover, and bone loss in elderly women. J Bone Miner Res. 1998;13(2):168-74.

2. Bischoff-Ferrari HA, Dietrich T, Orav EJ, Dawson-Hughes B. Positive association between 25-hydroxy vitamin D levels and bone mineral density: a population-based study of younger and older adults. Am J Med. 2004; 116(9):634-9.

3. Boyle WJ, Simonet WS, Lacey DL. Osteoclast differentiation and activation. Nature. 2003;423(6937):337-42.
4. Das S, Crockett JC. Osteoporosis - a current view of pharmacological prevention and treatment. Drug Des Devel Ther. 2013;7:435-48.

5. Boyce BF, Rosenberg E, de Papp AE, Duong LT. The osteoclast, bone remodelling and treatment of metabolic bone disease. Eur J Clin Invest. 2012:42(12):1332-41

6. Shen $\mathrm{CL}$, von Bergen $\mathrm{V}$, Chyu MC, Jenkins MR, Mo H, Chen $\mathrm{CH}$, et al. Fruits and dietary phytochemicals in bone protection. Nutr Res. 2012; 32(12):897-910

7. Yang F, Tang DZ, Cui XJ, Holz JD, Bian Q, Shi Q, et al. Classic yin and yang tonic formula for osteopenia: study protocol for a randomized controlled trial. Trials. 2011;12:187.

8. Zhao G, Cai D, Dong S, Fan Y. Clinical observation on treatment with the kidney-tonifying prescription in 25 cases of postmenopausal osteoporosis. J Tradit Chin Med. 2003;23(2):103-5.

9. Bae KH. Medicinal plants of Korea. Seoul: Kyohaksa; 2000.

10. WHO. Medicinal Plants in Mongolia. Switzerland: World Health Organization Press; 2013.

11. Gonda R, Tomoda M, Shimizu N, Kanari M. Characterization of an acidic polysaccharide from the seeds of Malva verticillata stimulating the phagocytic activity of cells of the RES. Planta Med. 1990;56(1):73-6.

12. Tomoda M, Shimizu N, Gonda R, Kanari M, Yamada H, Hikino H. Anticomplementary and hypoglycemic activities of the glycans from the seeds of Malva verticillata. Planta Med. 1990;56(2):168-70.

13. Kim JA, Yang SY, Kang SJ, Kim YH. Verticilloside, a new daucosteryl derivative from the seeds of Malva verticillata. Nat Prod Sci. 2011;17(4):350-53.

14. Ju D, Liu M, Zhao H, Wang J. Mechanisms of "kidney governing bones" theory in traditional Chinese medicine. Front Med. 2014;8(3):389-93.

15. Leung PC, Siu WS. Herbal treatment for osteoporosis: a current review. J Tradit Complement Med. 2013;3(2):82-7.

16. Zhang W, Fujikawa T, Mizuno K, Ishida T, Ooi K, Hirata T, et al. Eucommia leaf extract (ELE) prevents OVX-induced osteoporosis and obesity in rats. Am J Chin Med. 2012:40(4):735-52

17. Liu YQ, Zhan LB, Liu T, Cheng MC, Liu XY, Xiao HB. Inhibitory effect of Ecliptae herba extract and its component wedelolactone on preosteoclastic proliferation and differentiation. J Ethnopharmacol. 2014;157:206-11.

18. Chan BC, Lee HY, Siu WS, Yip KH, Ko CH, Lau CB, et al. Suppression of mast cell activity contributes to the osteoprotective effect of an herbal formula containing Herba Epimedii, Fructus Ligustri Lucidi and Fructus Psoraleae. J Pharm Pharmacol. 2014;66(3):437-44.

19. Seo B, Kim M, Park J, Jung P, Lee E. The effects of Ojeoksan water extract by various decoction method on inhibition of pain and inflammation. J Appl Ori Med. 2002;2(1):33-9.

20. Kim T, Kim K, Lee SH, So HS, Lee J, Kim N, et al. Identification of LRRc17 as a negative regulator of receptor activator of NF-kappaB ligand (RANKL)induced osteoclast differentiation. J Biol Chem. 2009;284(22):15308-16.

21. Kim HN, Lee JH, Bae SC, Ryoo HM, Kim HH, Ha H, et al. Histone deacetylase inhibitor MS-275 stimulates bone formation in part by enhancing Dhx36mediated TNAP transcription. J Bone Miner Res. 2011;26(9):2161-73.

22. Negishi-Koga T, Takayanagi H. Ca2 + -NFATC1 signaling is an essential axis of osteoclast differentiation. Immunol Rev. 2009;231(1):241-56.

23. Miyamoto T. Regulators of osteoclast differentiation and cell-cell fusion. Keio Med. 2011;60(4):101-5.

24. Kim HJ, Hong JM, Yoon KA, Kim N, Cho DW, Choi JY, et al. Early growth response 2 negatively modulates osteoclast differentiation through upregulation of Id helix-loop-helix proteins. Bone. 2012;51(4):643-50.

25. Maurer T, Zimmermann G, Maurer S, Stegmaier S, Wagner C, Hansch GM. Inhibition of osteoclast generation: a novel function of the bone morphogenetic protein 7/osteogenic protein 1. Mediators Inflamm. 2012;2012:171209.

26. Leung P, Pickarski M, Zhuo Y, Masarachia PJ, Duong LT. The effects of the cathepsin K inhibitor odanacatib on osteoclastic bone resorption and vesicular trafficking. Bone. 2011;49(4):623-35.

27. Shimizu N, Tomoda M. Constituents of the seed of Malva verticillata. I. Structural features of the major neutral polysaccharide. Chem Pharm Bull (Tokyo). 1987;35(12):4981-4.

28. Shimizu N, Tomoda M. Constituents of the seed of Malva verticillata. II. Characterizationo of Two Novel Neutral Polysaccharide. Chem Pharm Bull (Tokyo). 1988;36(8):2778-83.

29. Yang LC, Lu TJ, Lin WC. The prebiotic arabinogalactan of Anoectochilus formosanus prevents ovariectomy-induced osteoporosis in mice. J Funct Foods. 2013;5(4):1642-53. 
30. Kishimoto T, Kaneko T, Ukai T, Yokoyama M, Ayon Haro R, Yoshinaga Y, et al. Peptidoglycan and lipopolysaccharide synergistically enhance bone resorption and osteoclastogenesis. J Periodontal Res. 2012;47(4):446-54.

31. Cornish J, MacGibbon A, Lin JM, Watson M, Callon KE, Tong PC, et al. Modulation of osteoclastogenesis by fatty acids. Endocrinology. 2008; 149(11):5688-95.

32. Ransjo M, Sahli J, Lie A. Expression of connexin 43 mRNA in microisolated murine osteoclasts and regulation of bone resorption in vitro by gap junction inhibitors. Biochem Biophys Res Commun. 2003;303(4):1179-85

33. Yan XT, Lee SH, Li W, Jang HD, Kim YH. Terpenes and sterols from the fruits of Prunus mume and their inhibitory effects on osteoclast differentiation by suppressing tartrate-resistant acid phosphatase activity. Arch Pharm Res. 2015;38(2):186-92.

34. Zhang H, Xing WW, Li YS, Zhu Z, Wu JZ, Zhang QY, et al. Effects of a traditional Chinese herbal preparation on osteoblasts and osteoclasts. Maturitas. 2008;61(4):334-9.

35. Zhao Y, Li J, Liu Y, Yu KQ, Zhang J, Chen XG. Gu Ling Pian, a traditional Chinese medicine, regulates function and OPG/RANKL synthesis of osteoblasts via the p38 MAPK pathway. J Pharm Pharmacol. 2007:59(8):1167-73.

36. Jeong JC, Kang SK, Youn CH, Jeong CW, Kim HM, Lee YC, et al. Inhibition of Drynariae Rhizoma extracts on bone resorption mediated by processing of cathepsin K in cultured mouse osteoclasts. Int Immunopharmacol. 2003; 3(12):1685-97.

\section{Submit your next manuscript to BioMed Central} and we will help you at every step:

- We accept pre-submission inquiries

- Our selector tool helps you to find the most relevant journal

- We provide round the clock customer support

- Convenient online submission

- Thorough peer review

- Inclusion in PubMed and all major indexing services

- Maximum visibility for your research

Submit your manuscript at www.biomedcentral.com/submit

C) Biomed Central 